\title{
SỬ DỤNG ẢNH VÊ TINH LANDSAT 8 ĐỂ TÍNH TOÁN MỘT SỐ THÔNG SỐ MÔI TRƯờNG KHU VỰC TỈNH BẮC NINH TRONG GIAI ĐOẠN 2013 - 2015 - 2017
}

\author{
VŨ DƯƠNG THỤY, NGUYẼ̃N TRƯỜNG SONN \\ Cục Viễn thám quốc gia
}

\section{Tóm tắt:}

Bài báo trình bày tóm tắt kết quả sủ dụng ảnh vệ tinh Landsat 8 để xác định một số thành phần môi truòng chính của tỉnh Bắc Ninh trong giai đoạn 2013 - 2015 - 2017. Sư dụng ảnh vệ tinh Landsat 8 chup trong các năm 2013, 2015 và 2017 với phuơng pháp nội suy và hồi quy tuyến tính để đánh giá chất lương nước mặt (tính toán 3 thông số BOD5, COD và TSS) và chất lương không khi (bụi PM10 và chỉ số không khí API). Kết quả nghiên cứu cho thấy múc độ ô nhiếm tại tỉnh Bắc Ninh tăng dần trong giai đoạn 2013 - 2015 - 2017. Việc ứng dụng nguồn tu liệu ảnh Landsat 8 miễn phi trong việc tính toán các chỉ số chất lượng nước và không khi cho hiệu quả cao và tiết kiệm chi phí.

\section{1. Đặt vấn đề}

Trong giai đoạn 2013 - 2015 - 2017, Bắc Ninh là tỉnh có tốc độ tăng trưởng kinh tế với chỉ số tổng sản phẩm trên địa bàn tỉnh GRDP (Gross Regional Domestic Product) khoảng 19\% cao nhất cả nước [3]. Trong giai đoạn này, cùng với sự phát triển kinh tế xã hội thì tỉnh Bắc Ninh cũng phải đối mặt với tình trạng gia tăng về ô nhiễm môi trường, trong đó nổi bật là ô nhiễm không khí và chất lượng nước mặt. Vì vậy, công tác giám sát và cảnh báo tình trạng ô nhiễm môi trường trở nên hết sức cấp thiết.

Trong những năm gần đây, kỹ thuật viễn thám và GIS đã được ứng dụng rộng rãi trong nghiên cứu về sự biến động của các thành phần tài nguyên và môi trường [1]. Nhất là, trong việc đánh giá diễn biến và dự báo xu hướng các thành phần môi trường chính chịu sự tác động của con người. Trên thế giới, trong lĩnh vực môi trường không khí và môi trường nước, công nghệ viễn thám được coi là giải pháp phản ánh thực trạng khách quan trên diện rộng. Công nghệ viễn thám đã được thế giới áp dụng từ nhiều năm trước, từ những năm 70-80 của kỷ nguyên trước, khi ảnh Landsat và Spot xuất hiện trên thị trường (Verdin
J. 1985; Lathrop R. G., Lillesand T. M., 1989). Bước sang những năm đầu của kỷ nguyên 21 , không chỉ những nước sở hữu vệ tinh mới ứng dụng vào giám sát chất lượng nước mặt mà nhiều nước khác cũng đã ứng dụng công nghệ viễn thám (Pasterkamp R., et al. 2000; Richie J. C., et al., 2003; Yuan-Fong Su, et al., 2008; Keiser M., et. al., 2008; Manchino G., et al., 2009). Ở Việt Nam, TS. Lương Chính Kế đã sử dụng tư liệu viễn thám độ phân giải cao Spot5 để giám sát chất lượng nước mặt và không khí tại các vùng kinh tế trọng điểm (2010).

Các nghiên cứu nêu ở trên hầu hết đều sử dụng tư liệu ảnh viễn thám thương mại có giá thành cao (Spot5, Worldview...), hoặc ảnh viễn thám miễn phí nhưng có độ phân giải thấp (Landsat TM, MODIS). Trong khi đó, ảnh vệ tinh Landsat 8 có ưu điểm nổi bật là cung cấp thông tin khách quan, phong phú (11 kênh phổ), với chu kỳ lặp lại 16 ngày và cho độ phân giải cao nhất là $15 \mathrm{~m}$ [4], đáp ứng yêu cầu trong việc tính toán một số thành phần môi trường của khu vực cấp vùng, cấp tỉnh. Ngoài ra, ảnh vệ tinh Landsat 8 còn được cung cấp miễn phí tại địa chỉ trang web: https://earthexplorer.usgs.gov/. Chính vì vậy, nhóm nghiên cứu đã sử dụng ảnh 
vệ tinh Landsat 8 để tiến hành phân tích và tính toán xác định một số thành phần môi trường chính của tỉnh Bắc Ninh trong giai đoạn 2013 2015 - 2017. Đây cũng là kết quả nghiên cứu ứng dụng của đề tài nghiên cứu khoa học cấp Bộ: "Nghiên cứu ưng dụng ảnh Viễn thám và GIS đề dụ báo, đánh giá diễn biến của một số vấn đề môi truoòng chính trong việc xây dụng, theo dõi và giám sát đánh giá môi truờng chiến lược của địa phwong".

\section{Phương pháp nghiên cứu}

\subsection{Tài liệu sử dụng}

- Ảnh vệ tinh Landsat 8 chụp các năm 2013, 2015 và 2017 khu vực tỉnh Bắc Ninh bao gồm 06 cảnh ảnh được thu thập tại trang web https://earthexplorer.usgs.gov/. Thông tin chi tiết thể hiện theo bảng 1 bên dưới.

Bảng 1: Thông tin chi tiết ảnh vệ tinh Landsat 8 khu vưc tỉnh Bắc Ninh

\begin{tabular}{|c|c|c|}
\hline STT & Số hiệu cảnh & Ngày chụp \\
\hline 1 & LC0812704520131116T1 & $16 / 11 / 2013$ \\
\hline 2 & LC0812604520131109T1 & $09 / 11 / 2013$ \\
\hline 3 & LC0812704520151122T1 & $22 / 11 / 2015$ \\
\hline 4 & LC0812604520151115T1 & $15 / 11 / 2015$ \\
\hline 5 & LC0812704520171111T1 & $11 / 11 / 2017$ \\
\hline 6 & LC0812604520171104T1 & $04 / 11 / 2017$ \\
\hline
\end{tabular}

- Bản đồ địa hình tỷ lệ 1:50.000 khu vực tỉnh Bắc Ninh bao gồm các mảnh F-48-68-B, F-4868-D, F-48-69-A, F-48-69-C, F-48-69-D, F-4881-A và F-48-81-B (Sơ đồ hình 1).

- Số liệu chất lượng môi trường không khí áp dụng theo Thông tư số 28/2011/TT-BTNMT bao gồm 40 điểm lấy mẫu khu vực tỉnh Bắc Ninh tại các thời điểm 2013, 2015 và 2017. Cụ thể, gồm các thông số là: khí lưu huỳnh dioxit $\mathrm{SO}_{2}$, khí ni tơ dioxit $\mathrm{NO}_{2}$ và bụi TSP, PM10.

- Số liệu chất lượng nước mặt bao gồm 19 điểm lấy mẫu của khu vực tỉnh Bắc Ninh tại thời điểm các năm 2013, 2015 và 2017 chỉ thực hiện
3 chỉ tiêu là: Tổng chất rắn lơ lửng (TSS); Nhu cầu ôxy hóa học (COD); Nhu cầu ôxy sinh hóa $\left(\mathrm{BOD}_{5}\right)$.

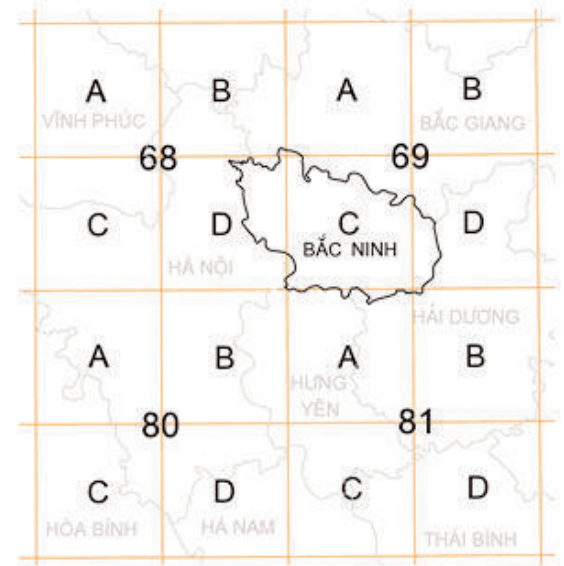

Hình 1: So đồ chia mảnh bản đồ địa hình tỷ lệ 1:50.000 khu vực tỉnh Bắc Ninh

\subsection{Phương pháp thụcc hiện}

\subsubsection{Phuoong pháp chuyển đổi tọa độ}

Ảnh vệ tinh Landsat $8 \mathrm{khu}$ vực nghiên cứu được tải về từ trang web https://earthexplorer.usgs.gov/ có hệ tọa độ UTM, elipxoid WGS84. Do đó, để có thể chồng lớp với $\mathrm{BĐĐH} \mathrm{tỷ} \mathrm{lệ} \mathrm{1:50.000,} \mathrm{ảnh} \mathrm{vệ} \mathrm{tinh}$ Landsat 8 sẽ được chuyển đổi tọa độ về hệ VN2000 bằng phương pháp tính chuyển sử dụng 7 tham số theo Quyết định số 05/2007/QĐBTNMT [2].

\subsubsection{Phương pháp xủ lý ảnh vệ tinh Landsat} 8

Để có kết quả tính toán khách quan, ảnh vệ tinh Landsat 8 được chuyển đổi từ giá trị cấp độ xám DN sang giá trị bức xạ phổ và được tiến hành hiệu chỉnh khí quyển [4].

- Chuyển đổi giá trị số (Digital Number) về giá trị bức xạ phổ (TOA Radiance) theo công thức:

$$
\mathbf{L}_{\chi}=\mathbf{M}_{\mathbf{L}} * \mathbf{Q}_{\mathrm{cal}}+\mathbf{A}_{\mathbf{L}}
$$

Trong đó:

$\mathrm{L}_{\chi}$ : giá trị bức xạ phổ tại ống kính của sensor 
$\left(\mathrm{Wm}^{-2} \operatorname{ster}^{-1} \mu \mathrm{m}^{-1}\right)$;

$\mathrm{Q}_{\mathrm{cal}}$ : giá trị số trên ảnh $(\mathrm{DN})$;

$\mathrm{M}_{\mathrm{L}}$ : giá trị RADIANCE_MULT_BAND_x được cung cấp từ file metadata;

$\mathrm{A}_{\mathrm{L}}$ : giá trị RADIANCE_ADD_BAND_x được cung cấp từ file metadata.

- Chuyển đổi giá trị giá trị số (Digital Number) về giá trị phản xạ phổ (TOA Reflectance) theo công thức:

$$
\rho_{\chi}=\left(M_{\rho} * Q_{\text {cal }}+A_{\rho}\right) / \cos \left(\boldsymbol{\theta}_{\text {sz }}\right)
$$

Trong đó:

$\rho_{\chi}$ : giá trị phản xạ phổ trên đỉnh khí quyển (Planetary TOA reflectancre);

$\mathrm{Q}_{\mathrm{cal}}$ : giá trị số trên ảnh $(\mathrm{DN})$;

$$
\mathrm{M}_{\rho} \text { : } \quad \text { giá }
$$

REFLECTANCRE_MULT_BAND_x được cung cấp từ file metadata;

$A_{\rho}:$ giá trị REFLECTANCRE _ADD_BAND_x được cung cấp từ file metadata;

$\Theta_{\mathrm{sz}}:$ góc thiên đỉnh.

- Sử dụng công cụ ATCOR trên phần mềm xử lý ảnh PCI Geomatica để tiến hành hiệu chỉnh khí quyển.

\subsubsection{Phương pháp phân tích hồi quy}

Dựa trên các kết quả nghiên cứu của [5] và [6], nhóm tác giả sử dụng các kênh phổ trong dải sóng nhìn thấy của ảnh vệ tinh Landsat 8 để thực hiện phân tích hồi quy.

Phương trình hồi quy có dạng tổng quát như sau:

$$
\mathrm{Y}=\mathrm{a}_{0}+\mathrm{a}_{1} * \mathrm{R} 1+\mathrm{a}_{2} * \mathrm{R} 2+\mathrm{a}_{3} * \mathrm{R} 3+\mathrm{a}_{4} * \mathrm{R} 4
$$

Trong đó:

Y là giá trị thông số môi trường cần xác định được xem là biến phụ thuộc;

Ri là giá trị ảnh sau hiệu chỉnh được xem là các biến độc lập với $\mathrm{R} 1$ là giá trị của kênh $\mathrm{B} 1$ (Coastal aerosol); R2 là giá trị của kênh B2 (Blue); R3 là giá trị của kênh $\mathrm{B} 3$ (Green) và R4 là giá trị của kênh $\mathrm{B} 4(\mathrm{Red})$;

$\mathrm{a}_{\mathrm{i}}(\mathrm{i}=0 \div 4)$ là các hệ số tương quan được xác định bằng thực nghiệm.

Cụ thể, phương trình hồi quy tính toán bụi PM10 trong các năm 2013, 2015 và 2017 có dạng như sau:

$$
\begin{gathered}
\mathrm{PM} 10_{2013}=194,79-1,07 * \mathrm{R} 1_{2013}- \\
3,71 * \mathrm{R} 2_{2013}+2,74 * \mathrm{R} 3_{2013}-0,53 * \mathrm{R} 4_{2013} \\
\mathrm{PM} 10_{2015}=189,94-20,45 * \mathrm{R} 1_{2015}+ \\
17,30 * \mathrm{R} 2_{2015}+3,38 * \mathrm{R} 3_{2015}-1,07 * \mathrm{R} 4_{2015} \\
\mathrm{PM} 10_{2017}=270,89-25,02 * \mathrm{R} 1_{2017}+ \\
10,45 * \mathrm{R} 2_{2017}+10,32 * \mathrm{R} 3_{2017}-1,37 * \mathrm{R} 4_{2017}
\end{gathered}
$$

Hệ số tương quan $\mathrm{R}^{2}$ của các năm 2013, 2015, 2017 lần lượt là $\mathrm{R}_{2013}^{2}=0,66 ; \mathrm{R}_{2015}^{2}=0,68$; $\mathrm{R}^{2}{ }_{2017}=0,66$. Kết quả này cho thấy mức độ phù hợp của mô hình là chấp nhận được trong việc tính toán phản ánh mức độ ô nhiễm bụi PM10 khu vực tỉnh Bắc Ninh.

Kết quả so sánh tương quan tuyến tính giữa giá trị đo ngoài thực địa và giá trị tính từ ảnh Landsat 8 thể hiện theo hình dưới đây: (Xem $\operatorname{hinh} 2)$

\subsubsection{Phương pháp nội suy}

Với 19 điểm đo chất lượng nước phân bố rải đều khu vực tỉnh Bắc Ninh, nhóm nghiên cứu lựa chọn sử dụng phương pháp nội suy IDW kết hợp với phương pháp viễn thám để tính toán các thông số BOD5, COD và TSS trong giai đoạn 2013 - 2015 - 2017.

\subsection{Kết quả đạt được}

- Kết quả tính toán một số thông số môi trường không khí bằng ảnh vệ tinh Landsat 8 khu vực tỉnh Bắc Ninh trong các năm 2013, 2015 và 2017 thể hiện trên các hình 3 dưới đây:

- Phân tích diễn biến ô nhiễm bụi PM10 trong giai đoạn 2013 - 2015 - 2017 (Hình 4) cho thấy 
chỉ số bụi PM10 tại khu vực tỉnh Bắc Ninh trong giai đoạn này đều vượt ngưỡng chất lượng không khí theo quy chuẩn QCVN 05:2013/BTNMT.

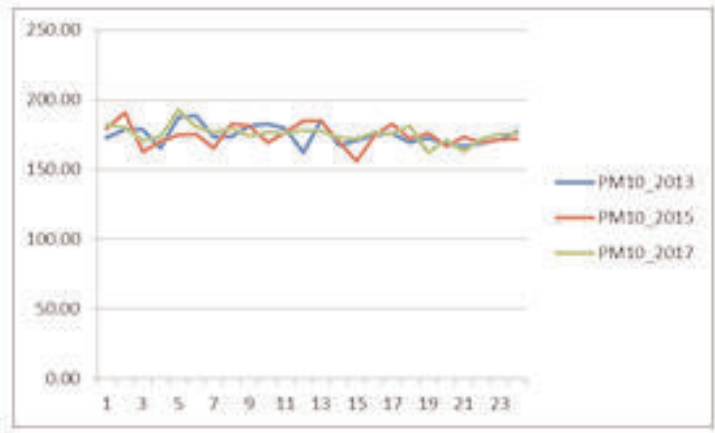

Hình 4: Diễn biến chi số bụi PM10 trong các năm 2013 - 2015 - 2017

- Kết quả tính toán một số thông số môi trường nước mặt bằng ảnh vệ tinh Landsat $8 \mathrm{khu}$ vực tỉnh Bắc Ninh trong các năm 2013, 2015 và 2017 thể hiện trên các hình dưới đây: (Xem hình $5,6,7)$

Căn cứ kết quả quan trắc tại tỉnh Bắc ninh 2017 cho thấy, chất lượng nước mặt trên địa bàn tỉnh Bắc Ninh tại một số vị trí lấy mẫu có hàm lượng các chất gây ô nhiễm vượt giá trị cho phép được quy định tại QCVN 08-MT:2015/BTNMT. Cụ thể như sau:

- Sông Ngũ Huyện Khê: pH dao động từ 6,5$7 ; \mathrm{BOD}_{5}$ dao động từ $9,7-2,162 \mathrm{mg} / \mathrm{l}$; $\mathrm{COD}$ từ 15 - 3,256 mg/l;. Lưu vực phía sau cầu Phong Khê là khu vực có hàm lượng các chất gây ô nhiễm cao nhất.

- Khu vực sông Cầu: $\mathrm{BOD}_{5}$ từ $<5$ - $54 \mathrm{mg} / \mathrm{l}$; COD từ $<3-105 \mathrm{mg} / 1 ; \mathrm{NH}_{4}$ từ $<0,005-0,27$ $\mathrm{mg} / \mathrm{l}$; Fe từ $<0,03-5,43 \mathrm{mg} / 1$; Photphat từ $<0,07$ -
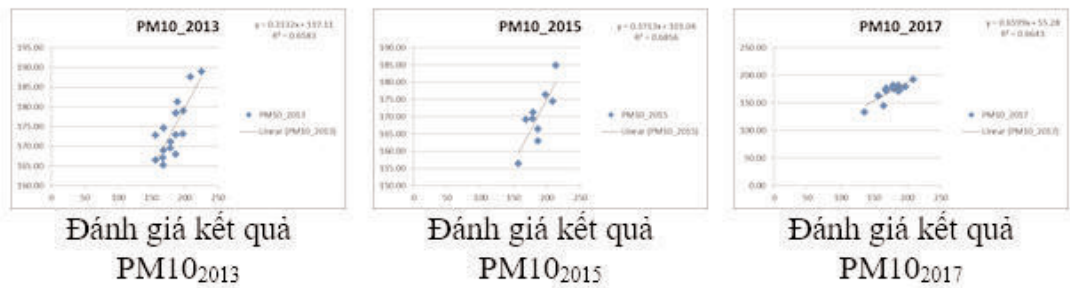

Hình 2: Đánh giá kết quả giá trị PM10 tính theo ảnh Landsat 8 với giá trị đo ngoài thực địa

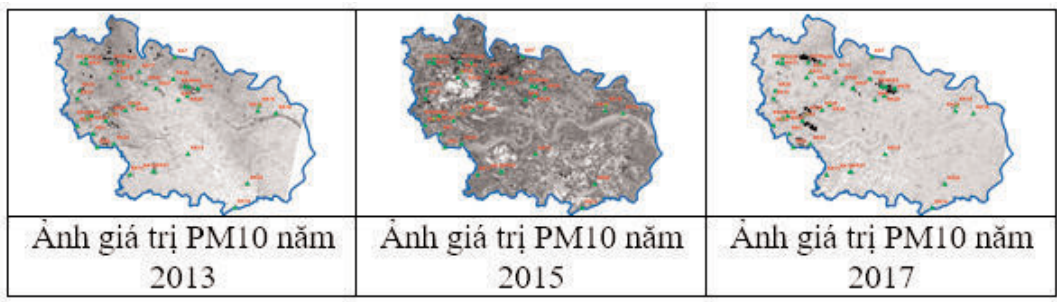

Hình 3: Kết quả tính toán giá trị PM10 theo ảnh Landsat 8

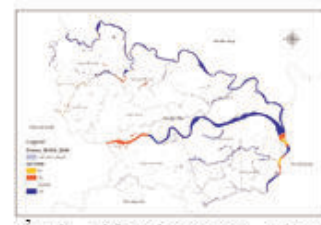

Ảnh giá trị $\mathrm{BOD}_{5}$ năm 2013

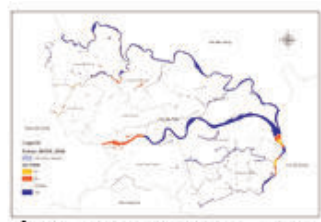

Ành giá trị $\mathrm{BOD}_{5}$ năm 2015

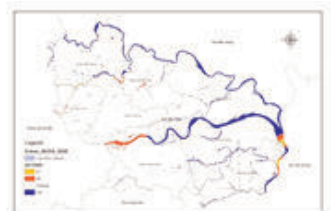

Ảnh giá trị $\mathrm{BOD}_{5}$ năm 2017

Hình 5: Kết quả tính toán giá trị $B O D_{5}$ theo ảnh Landsat 8 


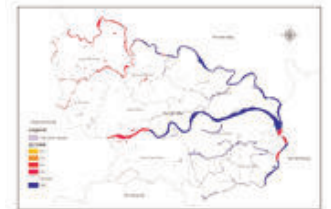

Ảnh giá trị COD năm 2013

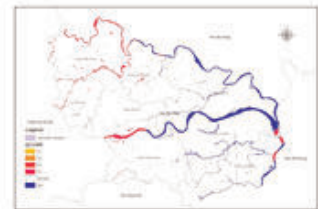

Ảnh giá trị COD năm 2015

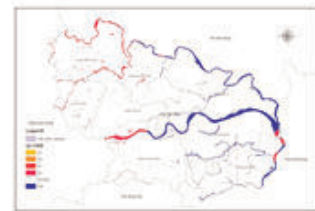

Ảnh giá trị COD năm 2017

Hình 6: Kết quả tính toán giá trị COD theo ảnh Landsat 8

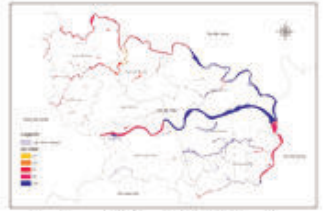

Ảnh giá trị TSS năm 2013

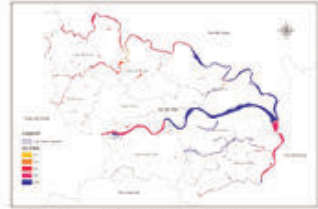

Ảnh giá trị TSS năm 2015

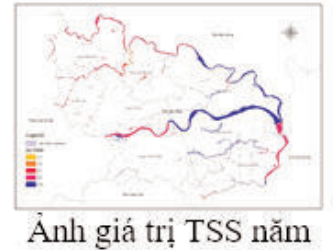

2017

Hình 7: Kết quả tính toán giá trị TSS theo ảnh Landsat 8

$5,55 \mathrm{mg} / \mathrm{l}$; Cr từ 0,054 mg/1;

- Khu vực sông Đuống: TSS từ $<5$ - 71,6 $\mathrm{mg} / \mathrm{l} ; \mathrm{BOD}_{5}$ dao động từ $<5-45 \mathrm{mg} / \mathrm{l} ; \mathrm{COD}$ từ 3 - $70 \mathrm{mg} / \mathrm{l}$;

- Khu vực sông Ngụ, sông Đồng Khởi, sông Bội, sông Khoai, sông Bắc Hưng Hải: TSS từ $<5$ - $102 \mathrm{mg} / 1 ; \mathrm{BOD}_{5}$ từ $<5$ - $40 \mathrm{mg} / \mathrm{l}$; COD từ $<3$ $158 \mathrm{mg} / \mathrm{l}$;

- Khu vực ngòi Tào Khê: TSS từ $<5$ - 142,8 $\mathrm{mg} / \mathrm{l} ; \mathrm{BOD}_{5}$ dao động từ $<5-28 \mathrm{mg} / \mathrm{l}$; $\mathrm{COD}$ từ $<3$ - $42 \mathrm{mg} / \mathrm{l}$;

- Nước mặt khu công nghiệp, cụm công nghiệp: TSS từ <5 - 150,6 mg/1; $\mathrm{BOD}_{5}$ dao động từ $<5$ - $220 \mathrm{mg} / \mathrm{l}$; COD từ $<3-93 \mathrm{mg} / \mathrm{l}$;

- Nước mặt khu vực làng nghề: TSS từ $<5$ $214,4 \mathrm{mg} / \mathrm{l} ; \mathrm{BOD}_{5}$ dao động từ $<5-175 \mathrm{mg} / \mathrm{l}$; COD từ $<3-320 \mathrm{mg} / 1$.

\section{Kết luận}

Ảnh vệ tinh Landsat 8 với độ phủ lớn $(180 \times 180 \mathrm{~km})$, sự đa dạng về kênh phổ và chu kỳ chụp lặp ngắn ngày (16 ngày) đã trở thành nguồn tư liệu quý giá trong công tác giám sát chất lượng môi trường không khí và chất lượng nước mặt khu vực tỉnh Bắc Ninh trong giai đoạn 2013 - 2015 - 2017.

Kết quả so sánh giá trị quan trắc mặt đất và giá trị phân tích ảnh cho thấy độ tin cậy của phương pháp viễn thám trong việc giám sát chất lượng môi trường trên một khu vực lớn.

Kết quả tính toán các thông số PM10 và chất lượng nước mặt $\mathrm{BOD}_{5}, \mathrm{COD}$, TSS tại tỉnh Bắc Ninh cho thấy xu hướng ô nhiễm ngày càng gia tăng, nhất là tại các khu công nghiệp và phát triển làng nghề. $\bigcirc$

\section{Tài liệu tham khảo}

[1]. Bộ Tài nguyên và Môi truoòng: Viễn thám Việt Nam: Khẳng định vai trò quan trọng trong công tác quản lý nhà nước về giám sát tài nguyên thiên nhiên và môi trường, phát triển kinh tế - xã hội, bảo đảm quốc phòng - an ninh, 2017.

[2]. Bộ Tài nguyên và Môi trường: Quyết định số 05/2007/QĐ-BTNMT về sử dụng hệ thống tham số tính chuyển giữa hệ tọa độ quốc tế WGS-84 và hệ tọa độ quốc gia VN-2000, 2007.

[3]. UBND tỉnh Bắc Ninh: Báo cáo phát triển kinh tế xã hội, 2013 - 2015 - 2017. 
[4]. USGS: Landsat 8 Data Users Handbook, 2019.

[5]. H. S. Lim, M. Z. MatJafri, K. Abdullah and $C$. J. Wong: Air pollution determination using remote sensing technique, 2009.
[6]. Cuc Viễn thám quốc gia: Úng dụng ảnh vệ tinh SPOT-5 thành lập bản đồ chất lượng nước mặt vùng cửa sông ven biển khu vực Quảng Ninh - Hải Phòng, 2014.O

\section{Summary}

Using landsat 8 satellite image data to assess calculate environmental parameters in Bac Ninh province in the period 2013 -2015 - 2017

Vu Duong Thuy, Nguyen Truong Son

\section{National Remote Sensing Department}

The paper summarizes the results of using Landsat 8 satellite images to identify some key environmental components of Bac Ninh province in the period 2013 - 2015 - 2017. Using Landsat 8 satellite images taken in 2013, 2015 and 2017 with linear interpolation and regression methods to assess surface water quality (calculating 3 parameters $\mathrm{BOD}_{5}, \mathrm{COD}$ and TSS) and air quality (PM10 dust and API air index). Research results show that pollution levels in Bac Ninh province gradually increase in the period 2013 - 2015 - 2017. The application of free Landsat 8 image sources in calculating water and air quality indicators for high efficiency and cost saving. $\bigcirc$

\section{NGHIÊN CÚ̉ PHÁT HIỆN, ĐÁNH GIÁ NHANH BIẾN ĐỘNG....}

(Tiếp theo trang 53)

\section{Summary}

Research to detect, rapid assessment of land cover change for updating geographic databases at scale 1:10.000

Pham Thi Hong Le, Pham Ngoc Phat, Tran Quang Minh

Department of Survey, Mapping and Geographic information Vietnam

Pham Ngoc Minh Anh

\section{National Economics University}

The paper presents the results of research and application of rapid and highly effective detection and assessment solutions on the amount, extent of land cover change serving the formulation of technical design - cost estimation of a project for updating the database using SPOT 6/7 images and the 2009 national geographic database. The authors convert the base geographic data from vector format to raster format combining classification, grouping, then use change detection technique based on multi-spectral image classification technique to create matrix of changes. The process of detecting and assessment of changes in geographical objects is carried out in Hung Tay commune, Hung Nguyen district, Nghe An province. $O$ 